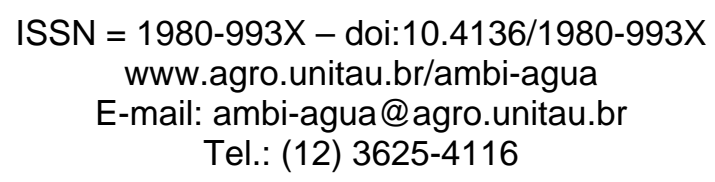

\title{
Avaliação de interpoladores para os parâmetros das equações de chuvas intensas no Espírito Santo
}

(doi:10.4136/ambi-agua.104)

\section{Roberto Avelino Cecilio ${ }^{1}$; Alexandre Cândido Xavier ${ }^{1}$; Fernando Falco Pruski ${ }^{2}$; Maycon Patrício de Hollanda ${ }^{3}$; José Eduardo Macedo Pezzopane ${ }^{1}$}

\author{
${ }^{1}$ Universidade Federal do Espírito Santo / Departamento de Engenharia Florestal \\ E-mail: racecilio@yahoo.com.br; xavier@cca.ufes.br; pezzopane2007@yahoo.com.br \\ ${ }^{2}$ Universidade Federal de Viçosa / Departamento de Engenharia Agrícola \\ E-mail: ffpruski@ufv.br \\ ${ }^{3}$ Universidade Federal do Espírito Santo \\ E-mail: mphollanda@hotmail.com
}

\section{RESUMO}

Chuvas intensas são muitas vezes responsáveis pela ocorrência de fenômenos indesejáveis em áreas agrícolas e florestais, tais como escoamento superficial, erosão hídrica e inundações. O conhecimento de sua distribuição espacial é, portanto, fundamental para o manejo de bacias hidrográficas, conservação do solo e dimensionamento de estruturas hidráulicas destinadas ao acúmulo ou transporte de água. O presente trabalho avaliou metodologias para interpolação dos parâmetros das equações de chuvas intensas ("K", "a", "b" e "c") para o Espírito Santo. Foram comparadas, para diferentes durações de chuva e períodos de retorno, as intensidades de precipitação reais com aquelas estimadas a partir dos valores interpolados dos parâmetros. Os resultados permitem concluir que o inverso da quinta potência da distância (IPD5) foi o método com melhor desempenho para a espacialização dos parâmetros "K", "a", "b" e "c".

Palavras-chave: espacialização; inverso de potência da distância; recursos hídricos; modelagem hidrológica; precipitação.

\section{Evaluation of intense rainfall parameters interpolation methods for the Espírito Santo State}

\begin{abstract}
Intense rainfalls are often responsible for the occurrence of undesirable processes in agricultural and forest areas, such as surface runoff, soil erosion and flooding. The knowledge of intense rainfall spatial distribution is important to agricultural watershed management, soil conservation and to the design of hydraulic structures. The present paper evaluated methods of spatial interpolation of the intense rainfall parameters ("K", "a", "b" and "c") for the Espírito Santo State, Brazil. Were compared real intense rainfall rates with those calculated by the interpolated intense rainfall parameters, considering different durations and return periods. Inverse distance to the $5^{\text {th }}$ power IPD5 was the spatial interpolation method with better performance to spatial interpolated intense rainfall parameters.
\end{abstract}

Keywords: spatialization; inverse distance to a power; water resources; hydrologic modeling; precipitation. 


\section{INTRODUÇÃO}

Chuvas intensas, também denominadas chuvas extremas ou máximas, são aquelas que apresentam grande lâmina precipitada, durante pequeno intervalo de tempo (Silva et al., 2003). Uma das características dessas chuvas é de possuírem distribuição irregular, tanto espacialmente quanto temporalmente (Araújo et al., 2008).

Por geralmente serem capazes de gerar grande quantidade de escoamento superficial, as precipitações intensas podem causar grandes prejuízos em áreas agrícolas, como a inundação de terras cultivadas, a erosão do solo, a perda de nutrientes, o assoreamento e a poluição de corpos d'água. Seu conhecimento é, portanto, fundamental para o planejamento de práticas de conservação do solo e da água, de manejo de bacias hidrográficas e para o dimensionamento de estruturas hidráulicas, tais como: barragens, vertedouros, terraços, drenos e bacias de contenção, dentre outros.

A caracterização das chuvas intensas é feita por intermédio de equações empíricas denominadas equações de intensidade-duração-frequência, ou equações de chuvas intensas (equação 1), derivada de dados pluviográficos de estações específicas. Portanto, essas equações são válidas somente para a estação da qual uma série de pluviogramas foi obtida.

$$
\mathrm{i}_{\mathrm{m}}=\frac{\mathrm{K} \mathrm{T}^{\mathrm{a}}}{(\mathrm{t}+\mathrm{b})^{\mathrm{c}}}
$$

em que:

$\mathrm{i}_{\mathrm{m}}$ é a intensidade de precipitação em $\mathrm{mm} \mathrm{h}^{-1}$; $\mathrm{T}$ é o período de retorno da precipitação em anos; $\mathrm{t}$ é a duração da precipitação em minutos; e $\mathrm{K}$, a, b, c são parâmetros de ajuste estatístico, referentes à localização do pluviógrafo para a qual a equação foi desenvolvida.

A determinação das equações de chuvas intensas apresenta grandes dificuldades em função da escassez de registros pluviográficos, da baixa densidade da rede pluviográfica e do pequeno período de observações disponível. Além disso, a metodologia para sua obtenção exige um exaustivo trabalho de tabulação, análise e interpretação de grande quantidade de pluviogramas (Oliveira et al., 2000).

Uma dificuldade frequentemente enfrentada pelos usuários dessas equações é a sua inexistência na localidade onde vai ser realizado um projeto qualquer. Essa dificuldade pode ser contornada utilizando-se a equação do pluviógrafo mais próximo, quando situado em região climática similar, ou se interpolando os parâmetros " $\mathrm{K}$ ", "a", "b" e "c" obtidos nas proximidades do local de interesse (Beltrame et al., 1991, Pruski et al., 2006).

Diversos trabalhos (Pruski et al., 1997 e 2006; Silva et al., 1999a e 1999b) sugerem a utilização de procedimentos de interpolação espacial para a determinação dos parâmetros da equação de chuvas intensas para qualquer localidade. Desses trabalhos, Pruski et al. (2006) utilizam o inverso da quinta potência da distância como interpolador, enquanto os demais utilizam o inverso do quadrado da distância. No entanto, Adamowski et al. (1996) destacam que vários métodos de interpolação desses parâmetros possuem limitações e são, frequentemente, inapropriados. Dessa forma, torna-se necessário avaliar a eficiência dos interpoladores antes de utilizá-los para espacializar os parâmetros das equações de chuvas intensas.

No intuito de prover melhores estimativas espaciais dos dados das equações de chuvas intensas, uma vez que o objeto de interesse é o valor da intensidade de precipitação $\left(\mathrm{i}_{\mathrm{m}}\right)$ e não dos parâmetros "K", "a", "b" e "c", é que Cecílio e Pruski (2003) propuseram realizar interpolações independentes para os parâmetros "K", "a", "b" e "c" e avaliar os resultados obtidos quando os valores interpolados são aplicados na equação 1. Todavia, os autores apenas avaliaram um único período de retorno $(\mathrm{T}=10$ anos $)$ e uma única duração de 
precipitação ( $\mathrm{t}=60$ minutos). Nessa mesma linha, Mello et al. (2008) avaliaram a continuidade espacial da intensidade de precipitação em Minas Gerais, a partir da utilização de três períodos de retorno e quatro durações da precipitação.

Do exposto, o presente trabalho teve por objetivos: a) avaliar diferentes métodos de interpolação dos parâmetros das equações de chuvas intensas para o Espírito Santo; b) comparar as intensidades de precipitação reais, considerando diferentes períodos de retorno $(2,5,10,20,50$ e 100 anos) e durações da precipitação $(10,20,30,40,50,60,120,240,360$, 720 e 1440 minutos), com aquelas estimadas a partir do uso dos parâmetros "K", "a", "b" e "c" interpolados.

\section{MATERIAL E MÉTODOS}

Utilizaram-se como base de dados, os parâmetros das equações de chuvas intensas de 20 estações pluviográficas do Estado do Espírito Santo (Figura 1), determinados por Silva et al. (1999a) e por Freitas et al. (2001). Criou-se um mapa representativo da localização de cada estação pluviográfica, associando-se a cada uma os valores dos parâmetros da equação de chuvas intensas ("K", "a", "b", “c").

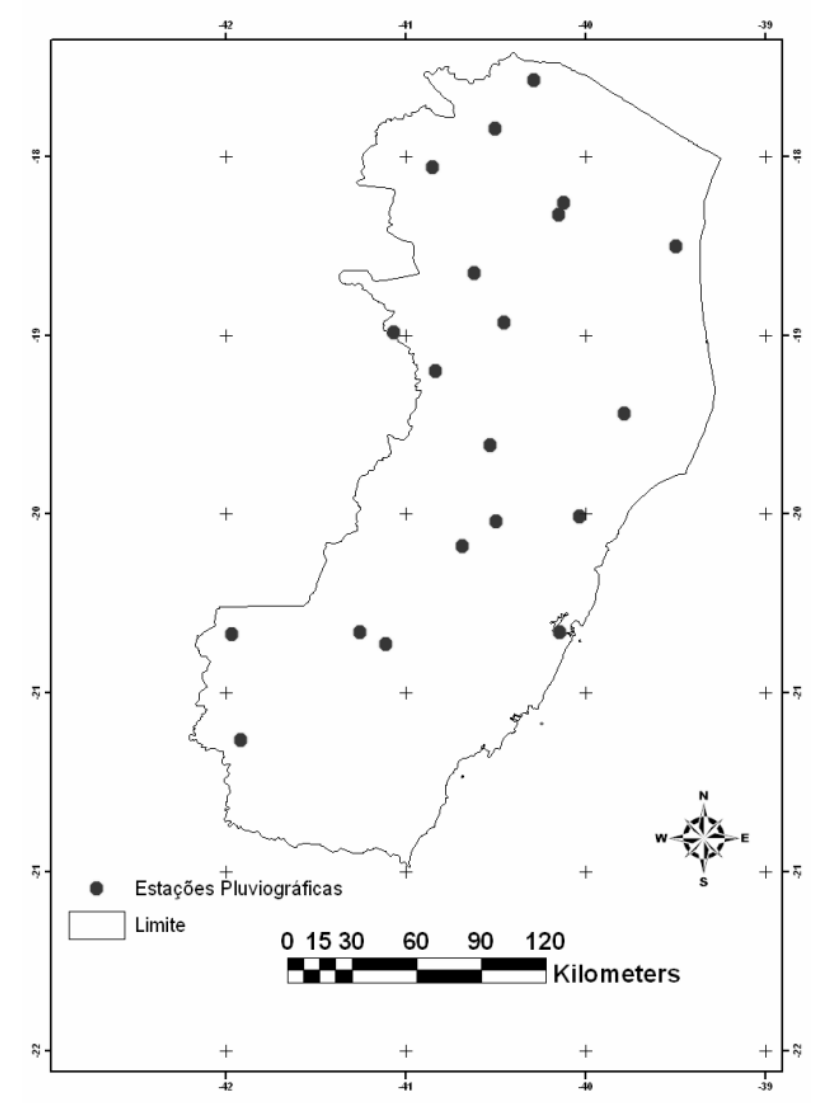

Figura 1. Localização das estações pluviográficas do estado do Espírito Santo utilizadas.

A análise dos métodos de interpolação foi feita com a utilização do método de validação cruzada, descrito por Myers (1997) e adaptado por Cecílio e Pruski (2003) e por Amorim et al. (2008). A partir da base de dados original, com a utilização do "software" Idrisi32 ${ }^{\circledR}$ procedeu-se a extração de uma das 20 estações previamente escolhidas, realizando-se, posteriormente a interpolação de cada um dos quatro parâmetros da equação de chuvas intensas, pelo método de interpolação do "Inverso de uma potência da distância" (IPD), utilizando-se quatro diferentes expoentes da distância: 2 (IPD2), 3 (IPD3), 4 (IPD4) e 5 
CECÍliO, R. A.; XAVIER,A. C.; PRUSKI, F. F.; HOLlANDA, M. P.; PEZZOPANE, J. E. M. Avaliação de interpoladores para os parâmetros das equações de chuvas intensas no Espírito Santo. Ambi-Agua, Taubaté, v. 4, n. 3, p. 82-92, 2009. (doi:10.4136/ambi-agua.104)

(IPD5). Após a realização da interpolação, a estação extraída era recolocada na base de dados e outra era escolhida para a extração. Esse procedimento foi realizado separadamente para cada uma das 20 estações pluviográficas apresentadas na Figura 1 e, dessa forma, foi possível comparar os valores reais e estimados dos quatro parâmetros da equação de chuvas intensas.

Convém ressaltar que, uma vez que a avaliação dos interpoladores é realizada com base em valores médios de índices estatísticos, quando se fixa determinado período de retorno, o erro na estimativa de $i_{m}$ pode ser muito baixo para determinadas durações e mais altos para outras, mascarando o resultado final. Dessa forma, torna-se necessário avaliar também a performance dos interpoladores a partir de valores fixos de duração da precipitação, com períodos de retorno diferentes.

Os valores interpolados (estimados) dos parâmetros "K", "a", "b" e "c" foram armazenados a fim de que, posteriormente e, para cada uma das 20 estações, fosse procedido o cálculo da intensidade de precipitação pela equação 1, utilizando-se os valores dos parâmetros interpolados por cada método. Para tanto, consideraram-se períodos de retorno (T) iguais a 2, 5, 10, 20, 50 e 100 anos, e durações da precipitação (t) iguais a 10, 20, 30, 40, 50, $60,120,240,360,720$ e 1440 minutos, resultando em 66 combinações diferentes.

Para cada uma das 66 combinações entre valores de $T$ e $t$, os valores de $i_{m}$ calculados $\left(E_{i}\right)$ foram confrontados com o valor de $\mathrm{i}_{\mathrm{m}}$ reais $\left(\mathrm{O}_{\mathrm{i}}\right)$, obtidos da aplicação dos valores originais de "K", "a", "b" e "c" (Silva et al., 1999a; Freitas et al., 2001) na equação 1.

A análise do desempenho do interpolador IPD foi feita a partir do cálculo de alguns índices estatísticos. Foram utilizados o índice de confiança (c) (Camargo e Sentelhas, 1997), o erro médio percentual (EMP) e o coeficiente de eficiência ajustado (E') (Legates e McCabe Jr., 1999), apresentados nas equações 2, 3 e 4, respectivamente.

$$
\begin{aligned}
& c=\left(1-\frac{\sum_{i=1}^{J}\left(O_{i}-E_{i}\right)^{2}}{\sum_{i=1}^{J}\left(\left|E_{i}-\bar{O}\right|+\left|O_{i}-\bar{O}\right|\right)^{2}}\right) r \\
& E M P=\frac{\sum_{i=1}^{J} \frac{\left|O_{i}-E_{i}\right|}{O_{i}}}{J} 100 \\
& E^{\prime}=1-\frac{\sum_{i=1}^{J}\left|O_{i}-E_{i}\right|}{\sum_{i=1}^{J}\left|O_{i}-\bar{O}\right|}
\end{aligned}
$$

em que:

J é o número de observações (no caso, estações em análise, ou seja, 20); $\mathrm{O}_{\mathrm{i}}$ é o valor real de intensidade de precipitação; $\mathrm{E}_{\mathrm{i}}$ é o valor de intensidade de precipitação calculado com o uso dos parâmetros interpolados; $\overline{\mathrm{O}}$ é a média dos valores reais de intensidade de precipitação; e $\mathrm{r}$ é o coeficiente de correlação entre os valores reais e estimados de intensidade de precipitação. Tabela 1.

O índice de confiança pode ser avaliado de acordo com a classificação apresentada na 
CECÍliO, R. A.; XAVIER,A. C.; PRUSKI, F. F.; HOLlANDA, M. P.; PEZZOPANE, J. E. M. Avaliação de interpoladores para os parâmetros das equações de chuvas intensas no Espírito Santo. Ambi-Agua, Taubaté, v. 4, n. 3, p. 82-92, 2009. (doi:10.4136/ambi-agua.104)

Tabela 1. Análise do desempenho dos interpoladores com base no índice de confiança (c).

\begin{tabular}{cc}
\hline Valor de c & Desempenho \\
\hline$>0,85$ & Ótimo \\
0,76 a 0,85 & Muito bom \\
0,66 a 0,75 & Bom \\
0,61 a 0,65 & Mediano \\
0,51 a 0,60 & Sofrível \\
0,41 a 0,50 & Mau \\
$\leq 0,40$ & Péssimo \\
\hline Fonte: Camargo e Sentelhas (1997) &
\end{tabular}

\section{RESULTADOS E DISCUSSÃO}

A Figura 2 mostra a variação dos valores dos índices de confiança (c) de Camargo e Sentelhas (1997) obtidos pelos diferentes métodos de interpolação em função da variação do período de retorno (Figura 2A) e em função da variação da duração da chuva intensa (Figura 2B).
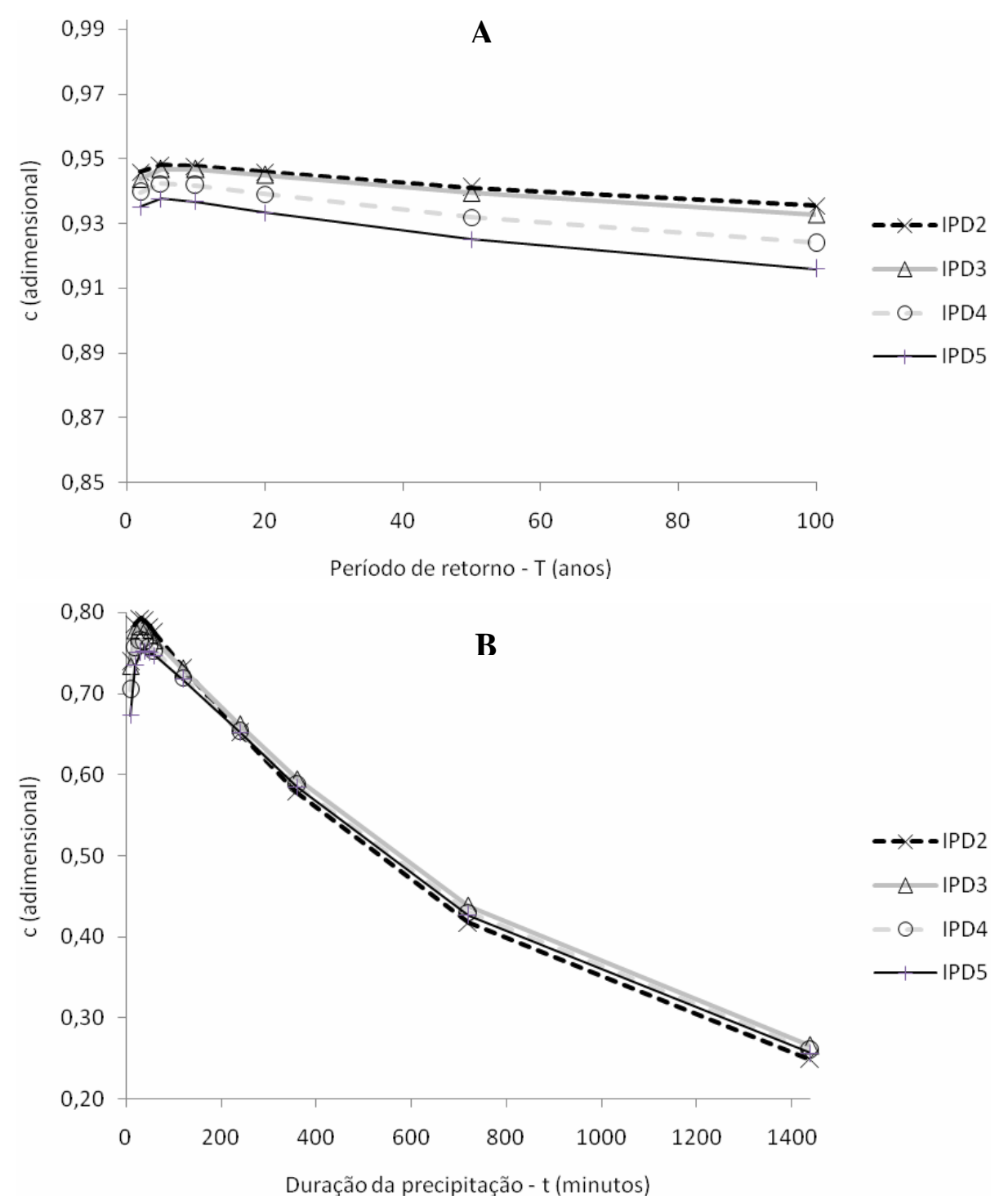

Figura 2. Valores dos índices de confiança (c) em função do período de retorno (A) e da duração da precipitação (B). 
CECÍlio, R. A.; XAVIER,A. C.; PRUSKI, F. F.; HOLlandA, M. P.; PEZZOPANE, J. E. M. Avaliação de interpoladores para os parâmetros das equações de chuvas intensas no Espírito Santo. Ambi-Agua, Taubaté, v. 4, n. 3, p. 82-92, 2009. (doi:10.4136/ambi-agua.104)

Observa-se que, com relação ao índice de confiança (c), os interpoladores avaliados obtiveram desempenho "ótimo" para os períodos de retorno considerados (Figura 2A e Tabela 1), embora, no geral, o valor de c para o IPD2 seja superior aos demais. A tendência geral observada é de melhor desempenho para o período de retorno de 5 anos, decrescendo o desempenho para maiores valores de T. Dessa forma, percebe-se que o período de retorno escolhido para a aplicação das equações de chuvas intensas pouco influencia na performance dos interpoladores, qualquer que seja a duração da precipitação escolhida.

Todavia, quando se analisam as durações da precipitação para períodos de retorno diferentes (Figura 2B e Tabela 1), observa-se que o desempenho foi "bom" ou superior apenas para as precipitações com menos de 240 minutos de duração. $\mathrm{O}$ valor do índice tende a diminuir com o aumento da duração da chuva até atingir a classificação péssima para as durações de 1440 minutos. Da mesma forma que analisado anteriormente, o desempenho dos interpoladores avaliados foi muito próximo, mas a superioridade numérica do índice coube ao IPD2 para as durações de até 120 minutos e ao IPD3 para as superiores.

A Figura 3 mostra a variação dos valores dos erros médios percentuais (EMP) obtidos pelos diferentes métodos de interpolação em função da variação do período de retorno (Figura 3A) e em função da variação da duração da chuva intensa (Figura 3B).

Comparando-se a influência dos períodos de retorno (Figura 3A), observa-se que o erro médio percentual (EMP) na estimativa de $\mathrm{i}_{\mathrm{m}}$ é mais alto para pequenos valores de $\mathrm{T}$ e tende a se estabilizar para os maiores valores de T. O método de interpolação capaz de propiciar o menor erro é o IPD5, variando entre $21,5 \%$ e $22,2 \%$. Dados publicados por Mello et al. (2003a), para o estado de São Paulo, permitem observar que os erros percentuais de estimativa a partir da aplicação do IPD2, considerando grandes períodos de retorno, praticamente não variam, corroborando os resultados aqui apresentados. Os dados do estudo de Mello et al. (2008) mostram a mesma tendência geral aqui observada, de aumento e estabilização do erro percentual com o aumento do período de retorno.

Pela análise da Figura 3B, também se observa os menores valores de EMP para o IPD5. Todavia, à medida que se aumentam as durações da precipitação, os erros médios de estimativa também são aumentados, variando entre 14,5\% e 42,5\%. Mello et al. (2003a) encontraram erros percentuais de estimativas em estações isoladas obtidos com a aplicação do IPD2, variando entre $4,4 \%$ e $27,8 \%$, havendo a tendência geral de aumento do erro percentual com o aumento da duração da precipitação, na maior parte das estações analisadas, corroborando a tendência observada no presente trabalho. $\mathrm{O}$ mesmo foi observado a partir dos dados publicados por Mello et al. (2008) para o estado de Minas Gerais, entretanto, os autores utilizaram o interpolador krigagem.

Cecílio e Pruski (2003) encontraram, para o estado de Minas Gerais, um valor de EMP, para o IPD5, igual a 19,37\%, considerando período de retorno de 10 anos e duração de precipitação de 60 minutos. Esse valor se encontra próximo àqueles encontrados no presente estudo para os mesmos valores de $\mathrm{T}$ e $\mathrm{t}$.

A análise dos resultados, apresentados na Figura 3, relativos ao EMP, corroboram aqueles relativos ao índice c (Figura 2), evidenciando por um lado a pouca influência do período de retorno sobre os erros de estimativa e, por outro, a grande influência da duração da precipitação sobre os erros obtidos. 
CECÍliO, R. A.; XAVIER,A. C.; PRUSKI, F. F.; HOLlANDA, M. P.; PEZZOPANE, J. E. M. Avaliação de interpoladores para os parâmetros das equações de chuvas intensas no Espírito Santo. Ambi-Agua, Taubaté, v. 4, n. 3, p. 82-92, 2009. (doi:10.4136/ambi-agua.104)
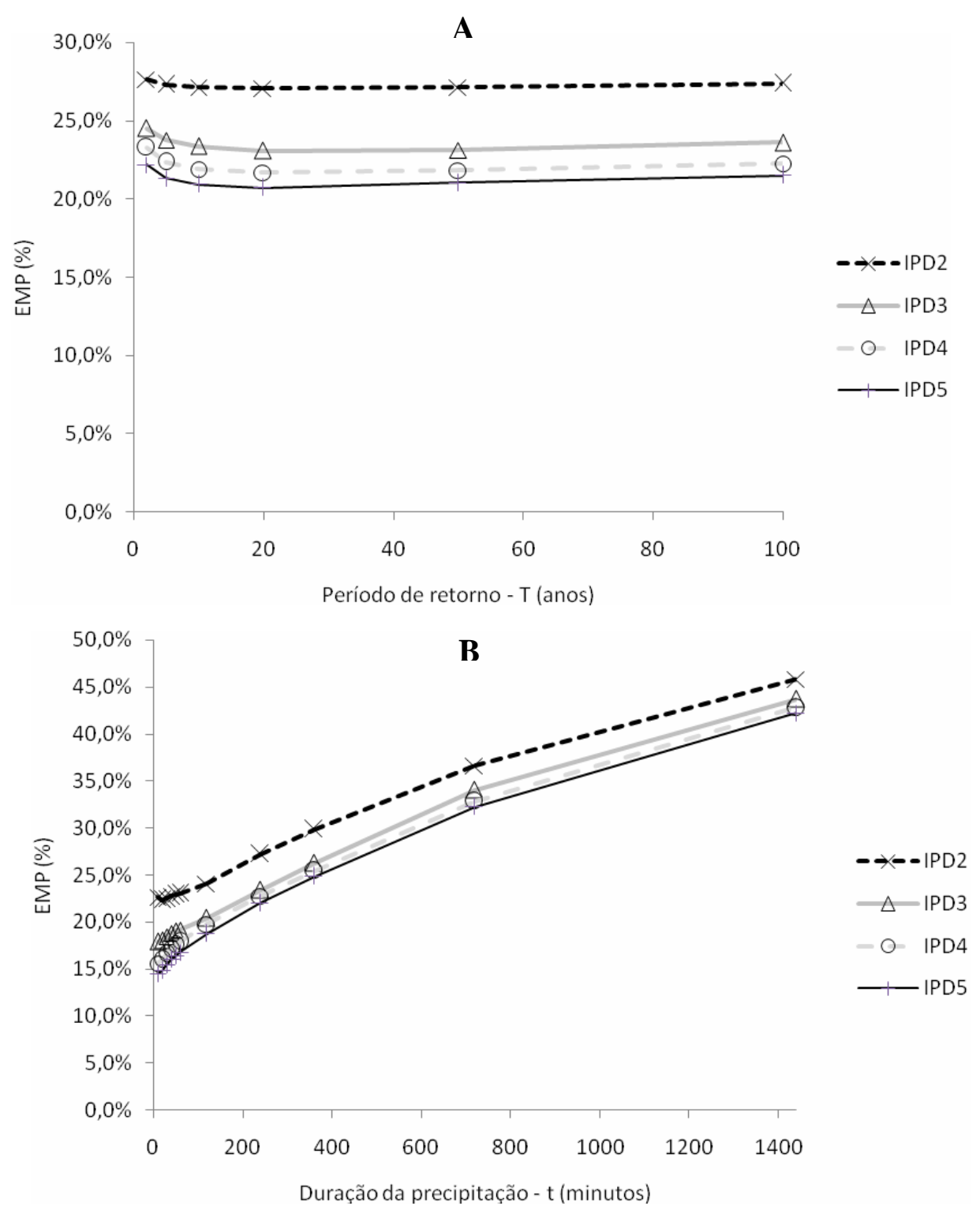

Figura 3. Valores do erro médio percentual (EMP) em função do período de retorno (A) e da duração da precipitação (B).

A Figura 4 mostra a variação dos valores dos índices de eficiência ajustados (E') obtidos pelos diferentes métodos de interpolação em função da variação do período de retorno (Figura 4A) e em função da variação da duração da chuva intensa (Figura 4B). 

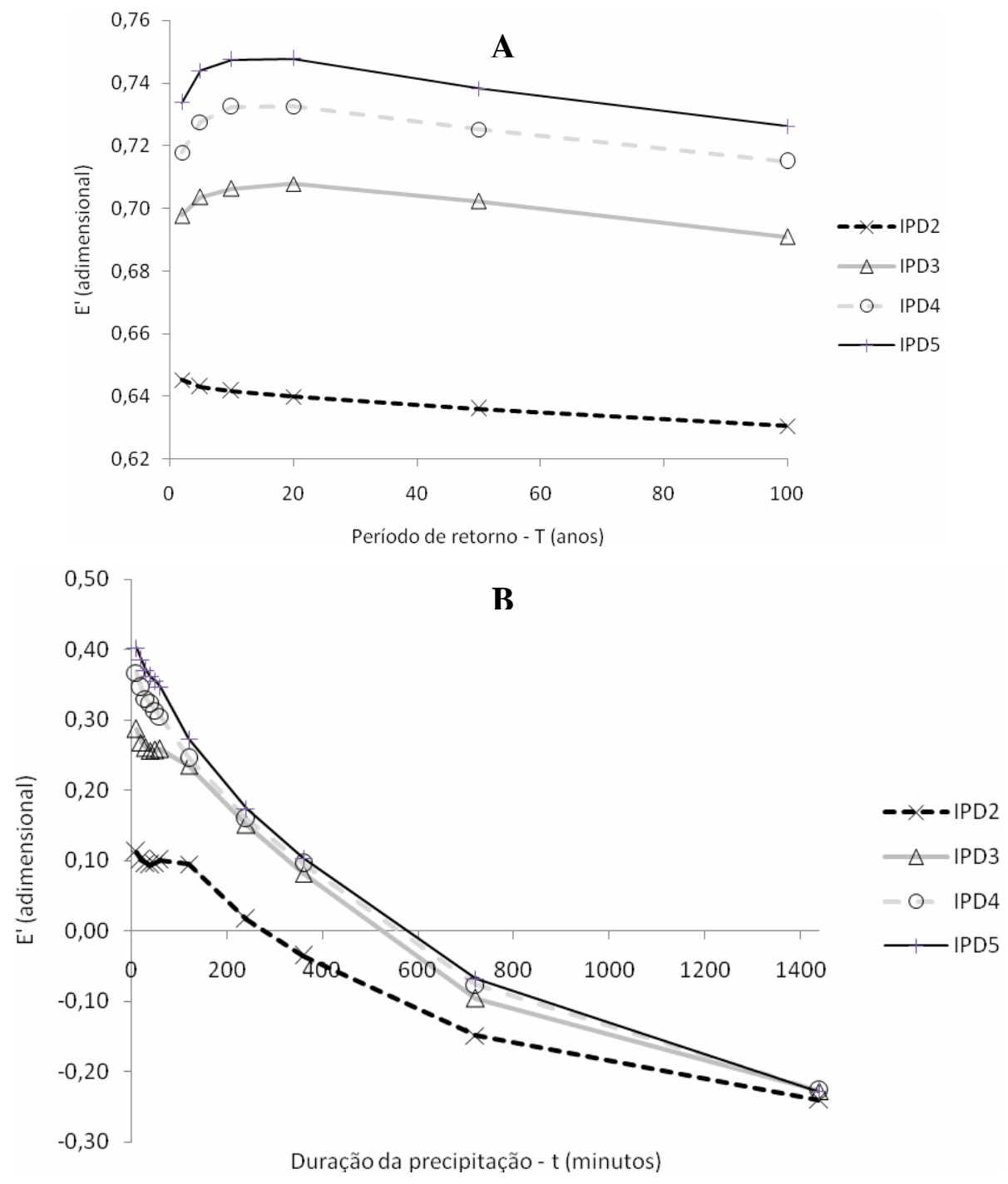

Figura 4. Valores do coeficiente de eficiência ajustado (E') em função do período de retorno (A) e da duração da precipitação (B).

O índice E' varia entre $-\infty$ e 1 , sendo que valores menores que zero acusam a ineficiência do método de estimativa, indicando que uma média simples dos valores seria mais representativa. Obviamente, quanto maior o valor do índice E', melhor a eficiência do método de estimativa.

Os maiores valores de E' foram obtidos, invariavelmente, pelo método IPD5 (Figura 5), embora os valores do IPD4 e do IPD3 fossem bastante próximos. O IPD2 acabou por apresentar os menores coeficientes de eficiência ajustado. $O$ aumento do expoente do interpolador IPD melhorou as interpolações, denotando que os postos mais próximos possuem maior influência nos valores dos parâmetros das equações de chuvas intensas.

Mello et al. (2003b) sugerem que o IPD2 é um método que apresenta imprecisões consideráveis na espacialização dos parâmetros das equações de chuvas intensas, fato corroborado no presente trabalho, devido aos baixos valores de E'.

Observa-se pela Figura 4A que, quando analisados os períodos de retorno, maiores valores foram obtidos quando o valor de $\mathrm{T}$ foi igual a 10 anos, diminuindo à medida que se afasta desse valor. Contudo, os valores variam pouco dentro dos diferentes períodos de retorno. 
Analisando-se a Figura 4B, é interessante verificar a influência que as durações da precipitação possuem sobre a magnitude do valor de E'. As baixas durações apresentaram os maiores valores de E', todavia estes foram inferiores a 0,5 . À medida que se aumentam as durações da precipitação, o coeficiente de eficiência ajustado tem seu valor drasticamente reduzido, chegando a ficar negativo, indicando que a interpolação passa a ser ineficiente. Conforme mostra a Figura 4B, para o IPD5 as durações de chuva acima de aproximadamente 590 minutos não podem ser bem estimadas a partir dos valores de K, a, b e c interpolados, sendo mais interessante usar a média dos valores de todas as estações do Espírito Santo para tal cálculo. O IPD2 começa a apresentar valores negativos para $t$ aproximadamente igual a 300 minutos.

De acordo com Chow et al. (1998), no meio rural, o dimensionamento de obras hidráulicas geralmente é realizado em bacias hidrográficas de pequeno porte, enquanto as chuvas intensas de projeto geralmente têm duração inferior a 120 minutos, sendo considerados períodos de retorno frequentemente inferiores a 20 anos. Nessa faixa de variação de $\mathrm{T}$ e $\mathrm{t}$, as interpolações realizadas pelo IPD5 apresentaram melhores eficiências, indicando aplicabilidade desse método de interpolação quando a finalidade das equações de chuvas intensas se restringir às aplicações realizadas em pequenas bacias hidrográficas localizadas em áreas rurais.

O IPD5 apresentou o melhor desempenho para a interpolação dos parâmetros das equações de chuvas intensas no Espírito Santo, corroborando os resultados obtidos por Cecílio e Pruski (2003) para Minas Gerais e, de certa forma validando o interpolador utilizado por Pruski et al. (2006) para todo o Brasil, no programa computacional Plúvio 2.1.

Mello et al. (2003a) sugerem que a interpolação dos parâmetros seja feita por meio da krigagem, pois esta apresenta menores erros percentuais de estimativa por ser baseada na condição de variância mínima e não tendência, reduzindo erros aleatórios por meio do controle de uma parcela desse erro, representada pela influência da posição das amostras (Mello et al., 2003b). Mello et al. (2008), encontraram, para Minas Gerais, erros médios percentuais no cálculo de $\mathrm{i}_{\mathrm{m}}$ variando entre $6,99 \%$ e $13,46 \%$ quando o método de interpolação utilizado foi a krigagem. Todavia, a aplicação da krigagem necessita de uma distribuição mais ou menos uniforme das estações pluviográficas (pontos amostrais), fato que não ocorreu no presente estudo.

\section{CONCLUSÕES}

Os resultados obtidos no presente trabalho permitem concluir que:

1. Dentre os interpoladores avaliados, o inverso da quinta potência da distância (IPD5) apresentou melhores resultados para espacialização dos parâmetros da equação de chuvas intensas no Espírito Santo.

2. Os interpoladores avaliados não apresentaram estimativas satisfatórias das intensidades de precipitação de chuvas intensas de longa duração.

3. A duração da precipitação possui maior influência sobre o desempenho da interpolação que o período de retorno.

\section{REFERÊNCIAS}

ADAMOWSKI, K.; ALILA, T.; PILON, P. J. Regional rainfall distribution for Canada. Atmospheric Research, v. 42, p. 75-88, 1996. 
CECÍlio, R. A.; XAVIER,A. C.; PRUSKI, F. F.; HOLlandA, M. P.; PEZZOPANE, J. E. M. Avaliação de interpoladores para os parâmetros das equações de chuvas intensas no Espírito Santo. Ambi-Agua, Taubaté, v. 4, n. 3, p. 82-92, 2009. (doi:10.4136/ambi-agua.104)

AMORIM, R. C. F.; RIBEIRO, A.; LEITE, C. C.; LEAL, B. G.; SILVA, J. B. G. Avaliação do desempenho dos métodos de espacialização da precipitação pluvial para o estado de Alagoas. Acta Scientiarum Technology, Maringá, v. 30, n. 1, p. 87-91, 2008.

ARAÚJO, L. E.; SOUSA, F. A. S.; RIBEIRO, M. A. F. M.; SANTOS, A. S.; MEDEIROS, P. C. Análise estatística de chuvas intensas na bacia hidrográfica do rio Paraíba. Revista Brasileira de Meteorologia, v. 23, n. 2, p. 162-169, 2008.

BELTRAME, L. F. S.; LANA, A. E. L.; LOUZADA, J. A. S. Chuvas intensas. Porto Alegre: IPH, UFRGS, 1991.69p.

CAMARGO, A. P.; SENTELHAS, P. C. Avaliação do desempenho de diferentes métodos de estimativa da evapotranspiração potencial no Estado de São Paulo. Revista Brasileira de Agrometeorologia, v. 5, p. 89-97, 1997.

CECÍLIO, R. A.; PRUSKI, F. F. Interpolação dos parâmetros da equação de chuvas intensas com uso do inverso de potências da distância. Revista Brasileira de Engenharia Agrícola e Ambiental, Campina Grande, v. 7, n. 3, p. 501-504, 2003.

CHOW, V. T.; MAIDMENT, D. R.; MAYS, L. W. Applied hydrology. New Yoork: McGraw-Hill, 1998. 572p.

FREITAS, A. J.; SILVA, D. D.; PRUSKI, F. F.; PINTO, F. A.; PEREIRA, S. B.; GOMES FILHO, R. R.; TEIXEIRA, A. F.; BAENA, L. G. N.; MELLO, L. T. A.; NOVAES, L. F. Equações de chuvas intensas no Estado de Minas Gerais. Belo Horizonte: Companhia de Saneamento de Minas Gerais; Viçosa: Universidade Federal de Viçosa, 2001. 65p.

LEGATES, D. R.; MCCABE JR., G. J. Evaluating the use of "goodness-of-fit" measures in hydrologic and hydroclimatic model validation. Water Resources Research, v. 35, n. 1, p. 233-241, 1999.

MELlO, C. R.; LIMA, J. M.; SILVA, A. M.; MELLO, J. M.; OLIVEIRA, M. S. Krigagem e inverso do quadrado da distância para interpolação dos parâmetros da equação de chuvas intensas. Revista Brasileira de Ciência do Solo, Viçosa, v. 27, n. 5, p. 925-933, 2003a.

MELLO, C. R.; SILVA, A. M., LIMA, J. M.; FERREIRA, D. F.; OLIVEIRA, M. S. Modelos matemáticos para predição da chuva de projeto para regiões do Estado de Minas Gerais. Revista Brasileira de Engenharia Agrícola e Ambiental, Campina Grande, v. 7, n. 1, p. 121-128, 2003b.

MELlO, C. R.; VIOLA, M. R.; MELLO, J. M.; SILVA, A. M. Continuidade espacial de chuvas intensas no estado de Minas Gerais. Ciência \& Agrotecnologia, Lavras, v. 32, n. 2, p. 532-539, 2008.

MYERS, J. C. Geostatistical error management: quantifying uncertainty for environmental sampling and mapping. New York: Van Nostrand Reinhold, 1997.

OLIVEIRA, L. F. C.; CORTÊS, F. C.; BARBOSA, F. O. A.; ROMÃO, P. A.; CARVALHO, D. F. Estimativa das equações de chuvas intensas para algumas localidades no estado de Goiás pelo método da desagregação de chuvas. Pesquisa Agropecuária Tropical, Goiânia, v. 30, n. 1, p. 23-27, 2000. 
CECÍliO, R. A.; XAVIER,A. C.; PRUSKI, F. F.; HOLlANDA, M. P.; PEZZOPANE, J. E. M. Avaliação de interpoladores para os parâmetros das equações de chuvas intensas no Espírito Santo. Ambi-Agua, Taubaté, v. 4, n. 3, p. 82-92, 2009. (doi:10.4136/ambi-agua.104)

PRUSKI, F. F.; CALIJURI, M. L.; BHERING, E. M.; SILVA, J. M. A. Metodologia baseada no uso de sistemas de informações geográficas para obtenção de equações de chuvas intensas em qualquer localidade do Estado do Paraná. Engenharia na Agricultura, Viçosa, v. 5, n. 3, p. 254-265, 1997.

PRUSKI, F. F.; TEIXEIRA, A. F.; SILVA, D. D.; CECÍlIO, R. A.; SILVA, J. M. A. Plúvio 2.1: chuvas intensas para o Brasil. In: PRUSKI, F. F.; SILVA, D. D.; TEIXEIRA, A. F.; CECÍlIO, R. A.; SILVA, J. M. A.; GRIEBELER, N. P. (Org.) HIDROS: dimensionamento de sistemas hidroagrícolas. Viçosa: UFV, 2006. p. 15-25.

SILVA, D. D.; PEREIRA, S. B.; PRUSKI, F. F.; GOMES FILHO, R. R.; LANA, A. M. Q.; BAENA, L. G. N. Equações de intensidade-duração-freqüência da precipitação pluvial para o estado de Tocantins. Engenharia na Agricultura, Viçosa, v. 11, n. 1/4, p. 7-14, 2003 .

SILVA, D. D.; PINTO, F. R. L.; PRUSKI, F. F.; PINTO, F. A. Estimativa e espacialização dos parâmetros da equação de intensidade-duração-freqüência da precipitação para 0 Rio de Janeiro e o Espírito Santo. Engenharia Agrícola, Jaboticabal, v. 18, n. 3, p. 1121, 1999a.

SILVA, D. D.; VALVERDE, A. E. L.; PRUSKI, F. F.; GONÇALVES, R. A. B. Estimativa e espacialização dos parâmetros da equação de intensidade-duração-freqüência da precipitação para o Estado de São Paulo. Engenharia Agrícola, Jaboticabal, v. 7, n. 2, p. 70-87, 1999 b. 